\title{
Reliability Analysis of Lithium Disilicate Crowns: Effect of Veneering and Milling Production Workflow
}

\author{
Cardelli, Paolo ; Serafini, Nicola ; Sinjari, Bruna ; Murmura, Giovanna ; Ȯzcan, Mutlu
}

DOI: https://doi.org/10.1111/jopr.12478

Posted at the Zurich Open Repository and Archive, University of Zurich ZORA URL: https://doi.org/10.5167/uzh-127850

Journal Article

Accepted Version

Originally published at:

Cardelli, Paolo; Serafini, Nicola; Sinjari, Bruna; Murmura, Giovanna; Ȯzcan, Mutlu (2016). Reliability Analysis of Lithium Disilicate Crowns: Effect of Veneering and Milling Production Workflow. Journal of Prosthodontics, 25(8):623-628.

DOI: https://doi.org/10.1111/jopr.12478 


\section{Category of manuscript: Short Communication}

\section{Reliability Analysis of Lithium Disilicate Crowns: Effect of Veneering and Milling Production Workflow}

Paolo Cardelli, DDS, ${ }^{1}$ Nicola Serafini DDS, ${ }^{1}$ Bruna Sinjari DDS PhD, ${ }^{1}$ Giovanna Murmura MD, DDS PhD, ${ }^{1}$ Mutlu Özcan, DDS, DMD, $\mathrm{PhD}^{2}$

1'University "G. D’Annunzio" of Chieti-Pescara, Department of Medical, Oral and Biotechnological Sciences, Chieti, Italy

${ }^{2}$ University of Zurich, Dental Materials Unit, Center for Dental and Oral Medicine, Clinic for Fixed and Removable Prosthodontics and Dental Materials Science, Zurich, Switzerland

Short title: Fracture resistance of lithium disilicate crowns

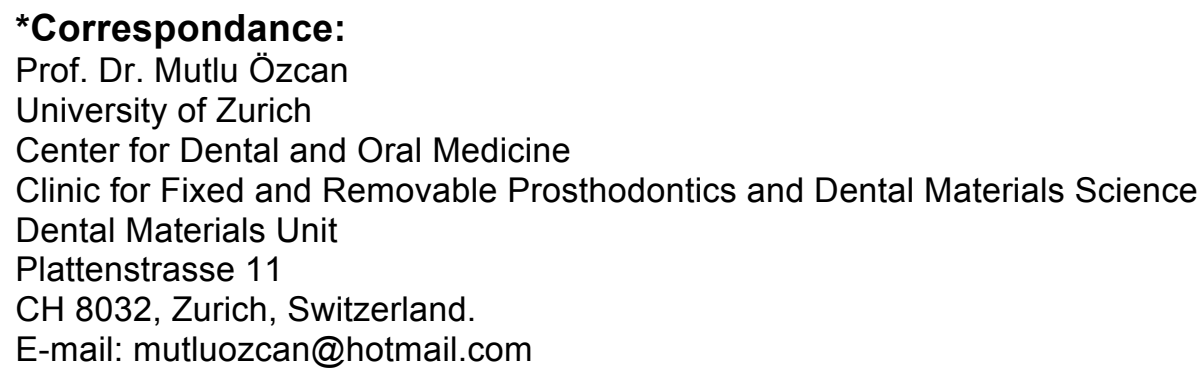




\section{Abstract}

Purpose: The aim for this study was to evaluate the reliability of lithium disilicate crowns produced using either CAD/CAM milling or thermopress workflow, for monolithic or veneered framework design for crowns.

Materials and Methods: Lithium disilicate crowns (e.max Press, Ivoclar Vivadent) $(\mathrm{N}=40)$ were produced using one of the four different workflows, namely a) MTP: Monolithic thermopressed crowns, b) MCM: Monolithic CAD/CAM milled, c) VTP: Thermopressed frameworks veneered or d) VCM: CAD/CAM milled crowns veneered. The specimens were adhesively luted (Variolink II, Ivoclar Vivadent) to resin composite abutments and then tested until fracture in a Universal Testing Machine $(1 \mathrm{~mm} / \mathrm{min})$. Failure types were classified and further evaluated under stereomicroscope and SEM. The data $(\mathrm{N})$ were analyzed using 1way ANOVA. Weibull distribution values including the Weibull modulus $(m)$, characteristic strength $(0)$, and probability of failure at $5 \%(0.05)$ were calculated.

Results: Fabrication method did not significantly influence the mean fracture strength ( $\mathrm{N}$ ) of lithium

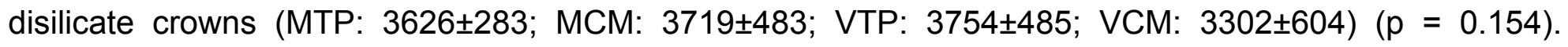
Weibull distribution presented lower shape value $(m)$ of VCM $(m=5.81)$ followed by VTP $(m=8.83), \mathrm{MCM}$ ( $m=8.84)$ compared to MTP $(m=12.9)$. Type $\vee$ failures (severe fracture of the crown and/or tooth) were experienced explicitly in all groups. The monolithic specimens showed a homogeneous structure without porosities. Wake hackles were observed at the fracture surface, with a radial path directed to the cervical area. The veneered crowns presented twist and wake hackles propagated perpendicularly from the cement-ceramic interface.

Conclusion: Monolithic or veneered lithium disilicate crowns presented similar fracture strength when frameworks or entire crowns were milled or thermopressed but Weibull characteristics was more favorable for monolithic pressed crowns and the least reliable for the milled and veneered crowns.

Clinical Significance: Lithium disilicate ceramic crowns could be produced either by thermopressing or CAD/CAM milling workflow without sacrificing from their reliability.

Keywords Adhesion; All-ceramic; CAD/CAM; Fracture strength; Lithium disilicate ceramic; Load-bearing capacity. 


\section{Introduction}

Metal-ceramic crowns still represent the gold standard for single-tooth prosthetic rehabilitation, with a 5year survival rate of $95.6 \% .{ }^{1}$ During the last few decades, increasing aesthetic demands and expectations of the patients for metal-free reconstructions led to the introduction of all-ceramic materials that are either polycrystalline or glass-based. ${ }^{2}$ The most common examples of polycrystalline ceramics are alumina and zirconia used for the fabrication of frameworks $\mathrm{s}^{3,4}$ where the latter has been used recently to produce full contour monolithic reconstructions for tooth- and implant-borne reconstructions. ${ }^{5}$ On the other hand, the glass-based reconstruction solutions, represented by feldspathic porcelain, leucite- or lithium disilicatereinforced ceramics, are suggested for their favourable optical properties. ${ }^{6}$ Among glass matrix ceramics, lithium disilicate reinforcement with $70 \%$ particles, ${ }^{7}$ having biaxial flexural strength of $400 \mathrm{MPa}$, is considered an adequate compromise for optical and mechanical properties. ${ }^{8}$ Lithium disilicate crowns can be fabricated through milling process using the CAD/CAM procedures or thermopressing workflow. The CAD/CAM workflow is based on the milling of blocks in partially crystallized phase that needs to be sintered in order to obtain the final mechanical properties. ${ }^{9}$ Thermopressed lithium disilicate restorations on the other hand, are obtained using a lost-wax technique, with ingots having the desired final shade and mechanical properties. Both CAD/CAM milling and thermopressed workflows can be used to construct a full contour monolithic crown or alternatively the framework could be subsequently veneered with the corresponding ceramic. ${ }^{10}$

The clinical performance of lithium disilicate single crowns has been investigated with a maximum followup of 8 years with the cumulative survival rate of $94.8 \% .{ }^{11}$ Mechanical properties of lithium disilicate crowns showed favourable results compared to metal-ceramic or zirconia crowns. However, the main failure type for lithium disilicate is characterized as fracture, and for metal-ceramic and zirconia, chipping of the veneering ceramic. ${ }^{12}$ In fact, monolithic crowns could be a solution to delamination or chipping problem experienced in bilayered reconstructions. However, studies comparing the mechanical properties of lithium disilicate crowns that are produced using either the milled or thermopressed workflows with and without 
veneering are lacking. Hence, comparing both workflows and crown designs in a single investigation could improve the knowledge about the performance of crowns made of lithium disilicate ceramic.

The objective of this study therefore was to evaluate the reliability of lithium disilicate crowns produced using either the CAD/CAM milling or thermopressing technique, with a monolithic design or a veneered framework. The null hypothesis tested was that the production workflow would not affect the fracture strength of lithium disilicate crowns.

\section{Materials and Methods}

Preparation of specimens

A maxillary first premolar tooth was prepared for a full crown restoration. The preparation comprised 1.5 $\mathrm{mm}$ axial, $2 \mathrm{~mm}$ occlusal reduction with bevel on the functional cusp and a deep chamfer finish line with rounded internal angles according to the manufacturer's recommendations. Ten impressions of the prepared master tooth were made using a two-phase polyvinylsiloxane impression material (Virtual, Ivoclar Vivadent, Schaan, Liechtenstein). The tooth abutments ( $N=40, n=10$ per group) were fabricated as described elsewhere, ${ }^{13}$ and impressions were filled with $2 \mathrm{~mm}$ layers of resin composite (Tetric EvoCeram Bulk Fill, Ivoclar Vivadent). One dental technician prepared the replicas under microscope (x20 magnification) (OPMI Movena, Carl Zeiss, Oberkochen, Germany) to ensure the absence of voids. They were then photo-polymerized (Optilux 501, Kerr Corp, Orange, CA, USA). The replicas were incubated in distilled water at $37^{\circ} \mathrm{C}$ for at least 30 days, allowing water hydration and avoiding any dimensional alteration of water-uptake expansion after crown cementation. ${ }^{14}$

Fabrication of monolithic CAD/CAM crowns (MCM)

An impression of the prepared master tooth was made with polyvinylsiloxane impression material (Virtual, Ivoclar Vivadent). A polyurethane resin replica of the master tooth (Exakto-Form, Bredent GmbH \& Co.KG, Senden, Germany) was produced only for scanning and design purposes similar to the clinical workflow and sent to a CAD/CAM milling centre. Then, an anatomical 3D CAD premolar crown was designed and 
milled with a five-axis CAM system from non-crystallized blocks (IPS e.max CAD, Ivoclar Vivadent). The thickness of the frameworks was $1 \mathrm{~mm}$ at the axial, and $1.3 \mathrm{~mm}$ at the occlusal side. These dimensions were chosen to offer an adequate support to the veneering ceramic. The space provided for veneering was $0.5 \mathrm{~mm}$ at the axial and $0.7 \mathrm{~mm}$ at the occlusal surfaces. Thus, the final crown was $1.7 \mathrm{~mm}$ thick at the axial and $2 \mathrm{~mm}$ at the occlusal side. After the milling procedure, final crystallization (Programat EP 5000, Ivoclar Vivadent) of the crowns was performed according to the manufacturer's guidelines. Finally, the crowns were glazed (e.max Ceram Glaze Paste, Ivoclar Vivadent).

Fabrication of monolithic thermopressed crowns (MTP)

In this group, the crowns were fabricated using the lost-wax press technique. The same STereoLithography (STL) file generated for the MCM group was used for milling wax (Ceramill Wax, Amann Girrbach AG, Koblach, Austria) replicas $(n=10)$ of the monolithic crowns. The wax crowns were invested (IPS PressVest Speed, Ivoclar Vivadent) and subsequently, a preheating cycle (Magma, Renfert $\mathrm{GmbH}$, Hilzingen, Germany) was carried out to remove the wax at $870^{\circ} \mathrm{C}$ with a holding time of 60 minutes. The resultant void in the mould was finally filled with the pressable ceramic (IPS e.max Press LT A3, Ivoclar Vivadent) in the ceramic furnace (Programat EP 5000, Ivoclar Vivadent), according to the manufacturer's recommendations. Next, the crowns were divested, separated, and air-abraded with 120 $\mu \mathrm{m}$ glass beads at a pressure of 2 bar. The crowns were then glazed as in group MCM.

Fabrication of veneered CAD/CAM (VCM) and thermopressed (VTP) crowns

The frameworks were fabricated as described for MCM and MTP and veneered (IPS e. max Ceram; Ivoclar Vivadent) according to the manufacturer's recommendations. In order to reproduce the monolithic crowns precisely, three impressions (Virtual, Ivoclar Vivadent) of the monolithic CAD/CAM crown were made. The first was mounted on a verticulator and used as a mould to precisely reproduce the occlusal surfaces of the crown. The other two impressions were cut from mesial to distal and labial to palatal sides, respectively, serving as a mould to control the veneering of axial surfaces. Finally, the crowns were glazed as described for group MCM. 
Adhesive cementation

Prior to cementation, the fit of each crown was checked on the resin composite (Tetric EvoCeram Bulk Fill, Ivoclar Vivadent) abutments using a black fit-checker (Fit Checker Black, GC Europe, Leuven, Belgium) under x20 magnification to identify any areas of friction. The cementation surfaces of the crowns were etched with hydrofluoric acid 5\% (IPS Empress Gel, Ivoclar Vivadent) for 20 seconds. Silane coupling agent (Monobond Plus, Ivoclar Vivadent) was applied one coat and waited for its reaction for 5 minutes and then adhesive resin (Heliobond, Ivoclar Vivadent) was applied using a microbrush.

The abutments were air-abraded with alumina particles coated with silica (CoJet Sand, 3M ESPE, Seefeld, Germany) for 20 seconds (2.5 bar, distance: $10 \mathrm{~mm}$ ). One coat of silane (Monobond Plus) was applied, allowed to react for 5 minutes and then adhesive resin (Heliobond) was applied. The crowns were cemented to their corresponding abutments with dual-polymerized resin cement (Variolink II, Ivoclar Vivadent) under a constant static load of $50 \mathrm{~N}$. After the excess cement was removed, the specimens were polymerized with an LED polymerization device (Radii-Cal, SDI, Bayswater Victoria, USA) for 40 seconds at each direction from a distance of $2 \mathrm{~mm}$ (light intensity: $1.200 \mathrm{mw} / \mathrm{cm}^{2}$ ). The specimens were then stored in distilled water at $37^{\circ} \mathrm{C}$ for 7 days prior to testing.

Fracture tests and failure analysis

The abutment/crown assemblies were embedded in acrylic resin (Orthocryl, Dentaurum GmbH \& Co. KG, Inspringen, Germany) using a modified parallelometer with the lowest point of the preparation margin 2 $\mathrm{mm}$ above the surface of the resin and the long axis of the crown-tooth perpendicular to the surface of the resin. The specimens were mounted in the Universal Testing Machine (LR30, Lloyd Materials Testing, Bognor Regis, UK) and subjected to single load-to-failure. Load was applied through an indenter (radius: $3.18 \mathrm{~mm}$ ) over the central fissure, at a cross-head speed of $1 \mathrm{~mm} / \mathrm{min}$ (Fig. 1). The maximum load was recorded in Newton, and the mean values were calculated for each group.

After fracture tests, failure types were intended to be categorized according to Burke`s classification: ${ }^{15,16}$ Type I: Cervical fracture or crack in the crown; Type II: Cohesive fracture not involving the framework or 
tooth; Type III: Cohesive fracture involving any interface; Type IV: Fracture involving the framework (root preserved); Type V: Fracture involving the root.

The most representative fractured specimens were further evaluated under stereomicroscope (OPMI Movena) at $\times 10$ to 80 and Scanning Electron Microscopy (SEM) (JSM-5500, JEOL Ltd., Akishima, Tokyo, Japan) at $\times 50$ to 100 magnification.

Statistical analysis

Statistical analysis was performed with SPSS Statistics for Windows Version 22 (SPSS, Chicago, IL, USA). Kolmogorov-Smirnov and Shapiro-Wilk tests were used to test normal distribution of the data. As the data $(\mathrm{N})$ were normally distributed, one-way analysis of variance (ANOVA) was used. Weibull analysis was performed using Minitab Version 14 (Minitab, State College, PA, USA). Weibull distribution values including the Weibull modulus $(m)$, characteristic strength $(0)$ and probability of failure at $5 \%(0.05)$, were calculated:

$$
\ln \ln \frac{1}{1-F_{\left(\sigma_{c}\right)}}=m \ln \sigma_{c}-m \ln \sigma_{0}
$$

\section{Results}

Fabrication workflow did not significantly influence the mean fracture strength $(\mathrm{N})$ of lithium disilicate crowns (MTP: 3626 \pm 283 ; MCM: 3719 \pm 483 ; VTP: 3754 \pm 485 ; VCM: 3302 \pm 604$)(p=0.154)($ Table 1). Weibull distribution presented lower shape value $(m)$ of VCM $(m=5.81)$ followed by VTP $(m=8.83)$, MCM ( $m=8.84)$ compared to MTP $(m=12.9)$ (Fig. 2).

Type $V$ failures (severe fracture of the crown and/or tooth) were experienced explicitly in all groups.

SEM findings revealed different fracture propagation patterns between monolithic and veneered crowns, regardless of the production method used. The monolithic specimens showed a homogeneous structure without porosities. Wake hackles were observed at the fracture surface, with a radial path directed to the cervical area (Fig. 3a). The veneered crowns did not show voids in the ceramic layering but twist and wake 
hackles propagated perpendicularly upwards from the cement-ceramic interface. Wallner lines showed their concave side pointing towards the beginning of the crack (Fig. 3b).

\section{Discussion}

This study was undertaken in order to compare the mechanical reliability of lithium disilicate crowns produced using either the CAD/CAM milling or thermopressing workflows. Based on the results obtained, since the workflow did not affect the results for monolithic and veneered crowns, the null hypothesis is rejected.

The fracture strength results obtained in this study ranged between 3302 and $3754 \mathrm{~N}$ being comparable to a previous report on monolithic CAD/CAM crowns $(3237 \mathrm{~N}){ }^{12}$ In another study, after fatigue conditions, the results decreased almost twofold ranging between 1618 and $1655 \mathrm{~N},{ }^{17}$ and $1719 \mathrm{~N} \cdot{ }^{18}$ All frameworks were made of lithium disilicate, having higher elasticity modulus $(95 \pm 5 \mathrm{GPa})^{10}$ compared to the veneering ceramic $(68 \mathrm{GPa}) .{ }^{20}$ Moreover, the Poisson ratio of lithium disilicate $(0.23)$ was similar to the veneering ceramic $(0.24){ }^{14,20}$ Materials with a high elasticity modulus tend to carry more of the load in a bilayered dental reconstruction. ${ }^{21}$ This could explain the similar behaviour of the four fabrication workflow in that less elastic framework sustain more of the load where the veneering ceramic follows the deformation pattern until final fracture.

In this study, resin composite used was abutment material instead of natural tooth that had an elasticity modulus of $15.5 \mathrm{GPa}$ and hardness of $0.73 \mathrm{GPa}$ comparable to that of human dentin 22,23 as it would be difficult to standardize the dimensions of the latter. ${ }^{17,24}$ Resin composite abutments were air-abraded in order to achieve micromechanical retention of the resin cement since adhesion of the cement to the abutment may play a role in fracture propagation at this interface. The situation in this experimental set up in terms of abutment material may clinically represent the situation where the crowns are cemented on the resin composite cores. The effect of abutment material type on the fracture strength of glass ceramic materials needs to be explored in future studies. 
Type $\mathrm{V}$ failures were observed exclusively in all groups which could be considered as catastrophic failures that cannot be repaired. Similarly, in a recent in vitro study, complete fractures but no chipping was observed for lithium disilicate crowns where the abutment material was natural tooth. ${ }^{25}$ These findings are supported in a clinical study in which findings of up to 4 years were reported where 1 fracture but no chippings were observed out of 29 single crowns. ${ }^{26}$ In another clinical study, out of 74 crowns, 5 fractures and 3 minor chippings of the veneering ceramic was observed after a mean observation period of 79.5 months. ${ }^{11}$ Such clinical failures indicate that lithium disilicate crowns are not problem-free up to an observation of 4 to 6.5 years. Unfortunately, information is lacking at this moment on the clinical survival of monolithic crowns.

Static loading tests has been criticized for not being clinically relevant. ${ }^{27}$ Yet, preclinical evaluations are still needed for ranking new fabrication concepts, methods or materials introduced in dentistry. In that respect, even though fracture strength values did not show significant difference, vast differences were observed in Weibull modulus between milled or thermopressed frameworks in both monolithic and veneered versions. Monolithic crowns fabricated through thermopressing (MTP, $m=12.9)$ showed considerably better reliability compared to milled versions ( $\mathrm{MCM}, m=8.84)$. This could be explained with the fact that milling burs create stress zones that eventually trigger crack propagation after fabrication of the crowns. Similarly, in the veneered versions of the crowns, veneering on milled crowns resulted in lower Weibull modulus (VCM, $m=5.81$ ) compared to veneering on thermopressed ones (VTP, $m=8.83$ ), again supporting the possible damage on the frameworks during milling procedures. These values are well above the moduli obtained for natural teeth when they are tested under static axial $(m=3.8)$ or lateral loading $(m=4.6) \cdot{ }^{28}$ The results of this study are slightly higher than those of a previous study where lithium disilicate crowns with knife edge $(m=5.48)$ and light chamfer type $(m=7.68)$ were tested on molars after subjecting the crowns to 1.200 .000 cycles. $^{17}$ One of the main causes of structural failure in dental reconstructions is the consequence of fatigue due to repeated stresses. Although static fracture tests may help to screen the reliability of reconstructions, cyclic loading could be considered a more clinically relevant 
testing approach. Yet, to date the parameters employed by the investigators such as the number of fatigue cycles, loading jigs, frequency of loading, presence of humid environment, involvement of hydrothermal aging conditions show a great variation in the current dental literature..$^{29}$ It has been reported that dental restorations fail more frequently under cyclic loading tests that are well below the ultimate flexural strength of these materials as opposed to the application of a single, relatively higher static load. However, no universal standard is presently available for such fatigue test methodologies.

The fractographic analysis confirmed the assumption that milling procedures create damage on the framework, namely the veneered specimens showed Wallner lines with their concave side pointing towards the beginning of the crack, showing fracture initiation at the loading point and the elastic behaviour of the veneering ceramic. However, the simultaneous loading transfer to the framework also allowed crack initiation from the framework-veneer interface with subsequent catastrophic failure. ${ }^{30}$ This behaviour was demonstrated by the position and direction of the wake and twist hackles. The fractographic analysis of monolithic specimens, either CAD/CAM milled or thermopressed, showed dominant radial crack pattern, starting from the loading area. This failure mode has been also demonstrated for monolithic ceramic structures previously. ${ }^{17,31}$

Clinical studies on the longevity of lithium disilicate crowns should report on the association between the incidences of failure and relate the results to the workflow employed. Further studies with cyclic loading and subsequent clinical evaluation are needed to finally validate this concept.

\section{Conclusions}

Monolithic or veneered lithium disilicate ceramic crowns presented no significant difference in fracture strength after the frameworks or entire crowns were milled or thermopressed. Yet, Weibull characteristics was more favorable for monolithic pressed crowns and the least reliable for the milled and veneered 
crowns. Accordingly, such ceramic crowns may better be produced by thermopressing workflow and do not necessitate veneering or milling for more reliability.

\section{Acklowledgements}

The study was entirely funded with departmental financial resources and performed with departmental devices.

\section{Conflict of interest}

The authors did not have any commercial interest in any of the materials used in this study. 


\section{References}

1. Raigrodski AJ, Hillstead MB, Meng GK, et al: Survival and complications of zirconia-based fixed dental prostheses: a systematic review. J Prosthet Dent 2012;107:170-177.

2. Guess PC, Schultheis S, Bonfante EA, et al: All-ceramic systems: Laboratory and clinical performance (Review). Dent Clin North Am 2011;55:333-352.

3. Wassermann A, Kaiser M, Strub JR: Clinical long-term results of VITA In-Ceram Classic crowns and fixed partial dentures: A systematic literature review (Review). Int J Prosthodont 2006;19:355-363.

4. Kelly JR, Benetti P: Ceramic materials in dentistry: Historical evolution and current practice (Review). Aust Dent J 2011;56:84-96.

5. Stober T, Bermejo JL, Rammelsberg P, et al: Enamel wear caused bymonolithic zirconia crowns after 6 months of clinical use. J Oral Rehabil 2014;41:314-322.

6. Zarone F, Russo S, Sorrentino R: From porcelain-fused-to-metal to zirconia: Clinical and experimental considerations. Dent Mater 2011;27:83-96.

7. Pollington S: Novel glass-ceramics for dental restorations. J Contemp DentPract 2011;12:60-67.

8. Ma L, Guess PC, Zhang Y: Load-bearing properties of minimal-invasive monolithic lithium disilicate and zirconia occlusal onlays: Finite element and theoretical analyses. Dent Mater 2013;29:742-751.

9. Kang SH, Chang J, Son HH: Flexural strength and microstructure of two lithium disilicate glass ceramics for CAD/CAM restoration in the dental clinic. Restor Dent Endod 2013;38:134-140.

10. Silva NR, Bonfante EA, Martins LM, et al: Reliability of reduced-thickness and thinly veneered lithium disilicate crowns. J Dent Res 2012;91:305-310.

11. Gehrt M, Wolfart S, Rafai N, et al: Clinical results of lithium-disilicate crowns after up to 9 years of service. Clin Oral Investig 2013;17:275-284. 
12. Silva NR, Thompson VP, Valverde GB, et al: Comparative reliability analyses of zirconium oxide and lithium disilicate restorations in vitro and in vivo. J Am Dent Assoc 2011;142:4-9.

13. Coelho PG, Bonfante EA, Silva NR, et al: Laboratory simulation of Y-TZP all-ceramic crown clinical failures. J Dent Res 2009;88:382-386.

14. Albakry M, Guazzato M, Swain MV: Biaxial flexural strength, elastic moduli, and x-ray diffraction characterization of three pressable all-ceramic materials. J Prosthet Dent 2003;89:374-380.

15. Burke FJ, Qualtrough AJ, Hale RW: The dentine-bonded ceramic crown: An ideal restoration? Br Dent J 1995;179:58-63.

16. Burke FJ: Fracture resistance of teeth restored with dentin-bonded crowns: The effect of increased tooth preparation. Quintessence Int 1996;27:115-121.

17. Cortellini D, Canale A, Souza RO, et al: Durability and Weibull characteristics of lithium disilicate crowns bonded on abutments with knife-edge and large chamfer finish lines after cyclic loading. $\mathrm{J}$ Prosthodont. 2014 (Epub).

18. Carvalho AO, Bruzi G, Giannini M et al: Fatigue resistance of CAD/CAM complete crowns with a simplified cementation process. J Prosthet Dent 2014

19. Ivoclar Vivadent. IPS e.max Scientific Documentation, 2006.

20. Ereifej N, Rodrigues FP, Silikas N, et al: Experimental and FE shear-bonding strength at core/veneer interfaces in bilayered ceramics. Dent Mater 2011;27:590-597.

21. Magne P, Belser UC: Porcelain versus composite inlays/onlays: Effects of mechanical loads on stress distribution, adhesion, and crown flexure. Int J Periodontics Restorative Dent 2003;23:543-555.

22. Watts DC, el Mowafy OM, Grant AA: Temperature-dependence of compressive properties of human dentin. J Dent Res 1987;66:29-32. 
23. El-Safty S, Akhtar R, Silikas N, et al: Nanomechanical properties of dental resin-composites. Dent Mater 2012;28:1292-1300.

24. Heintze SD, Albrecht T, Cavalleri A et al: A new method to test the fracture probability of all-ceramic crowns with a dual-axis chewing simulator. Dent Mater 2011;27:e10-e19.

25. Seydler B, Rues S, Müller D, et al: In vitro fracture load of monolithic lithium disilicate ceramic molar crowns with different wall thicknesses. Clin Oral Investig 2014;18:1165-1167.

26. Reich S, Schierz O: Chair-side generated posterior lithium disilicate crowns after 4 years. Clin Oral Investig 2013;17:1765-1772.

27. Kelly JR, Benetti $P$, Rungruanganunt $P$, et al: The slippery slope: critical perspectives on in vitro research methodologies. Dent Mater 2012;28:41-51.

28. Gresnigt M, Özcan M, van den Houten MLA, et al: Fracture strength, failure type and Weilbull characteristics of lithiumdisilicate and multiphase resin composite endocrowns under axial and lateral forces. Dent Mater 2015 (Epub).

29. Baran G, Boberick K, McCool J. Fatigue of restorative materials. Crit Rev Oral Biol Med 2001;12:350360 .

30. Lawn BR, Deng Y, Thompson VP: Use of contact testing in the characterization and design of allceramic crownlike layer structures: A review. J Prosthet Dent 2001;86:495-510.

31. Deng Y, Lawn BR, Lloyd IK: Characterization of damage modes in dental ceramic bilayer structures. J Biomed Mater Res 2002;63:137-145. 


\section{Captions to tables and legends:}

Tables:

Table 1. Fracture strength results (Mean \pm standard deviation) (Newton) of lithium disilicate crowns in experimental groups, minimum, maximum and Confidence Intervals (95\%). Same lower-case letters in each column indicate no significant differences ( $p>0.05)$. MTP: Monolithic thermopressed; MCM: Monolithic CAD/CAM milled; VTP: Thermopressed frameworks veneered; VCM: CAD/CAM milled crowns veneered.

\section{Figures:}

Figure 1 The position of the load cell in relation to the occlusal surface of the crowns in the universal testing machine where loading was applied until fracture.

Figure 2 Probability plot with Weibull curves $(95 \% \mathrm{Cl})$ using maximum likelihood estimation, scale and shape values for all groups. See Table 1 for group abbreviations.

Figures 3a-b SEM images of a) Monolithic lithium disilicate crown after fracture at $x 75$ from the occlusal surface of the veneered crown. The black arrows represent the location and direction of wake hackles and twist hackles. The well-defined concentric lines indicate arrested cracks, b) Veneered lithium disilicate crown after after fracture at $\times 89$. The black arrows indicate Wallner lines and white arrows represent the location and direction of wake and twist hackles. 
Tables:

\begin{tabular}{|c|c|c|c|c|c|c|}
\hline $\begin{array}{c}\text { Experimental } \\
\text { Groups }\end{array}$ & $\mathbf{n}$ & Mean (SD) & Minimum & \multicolumn{2}{|c|}{ Maximum } & \multicolumn{2}{|c|}{ Confidence Interval } \\
\cline { 3 - 6 } & 10 & $3626 \pm 283^{\mathrm{a}}$ & 3272 & 4222 & 3423 & 3828 \\
\hline MTP & 10 & $3719 \pm 483^{\mathrm{a}}$ & 3055 & 4556 & 3373 & 4064 \\
\hline MCM & 10 & $3754 \pm 485^{\mathrm{a}}$ & 3036 & 4599 & 3407 & 4101 \\
\hline VTP & 10 & $3302 \pm 604^{\mathrm{a}}$ & 2593 & 4554 & 2870 & 3734 \\
\hline VCM & & & & & & \\
\hline
\end{tabular}

Table 1. Fracture strength results (Mean \pm standard deviation) (Newton) of lithium disilicate crowns in experimental groups, minimum, maximum and Confidence Intervals (95\%). Same lower-case letters in each column indicate no significant differences $(p>0.05)$. MTP: Monolithic thermopressed; MCM: Monolithic CAD/CAM milled; VTP: Thermopressed frameworks veneered; VCM: CAD/CAM milled crowns veneered. 


\section{Figures:}

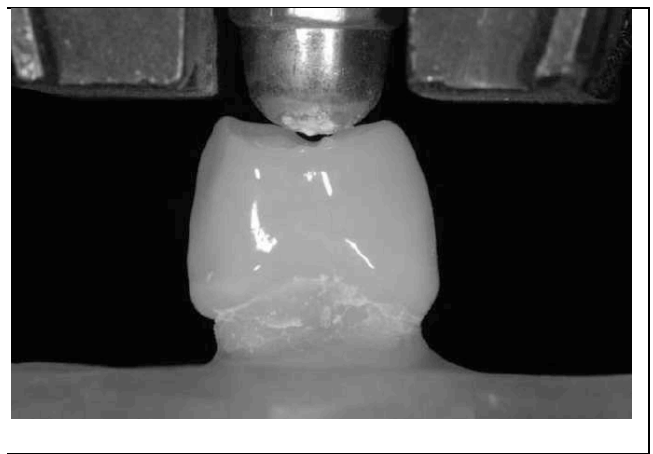

Figure 1 The position of the load cell in relation to the occlusal surface of the crowns in the universal testing machine where loading was applied until fracture.

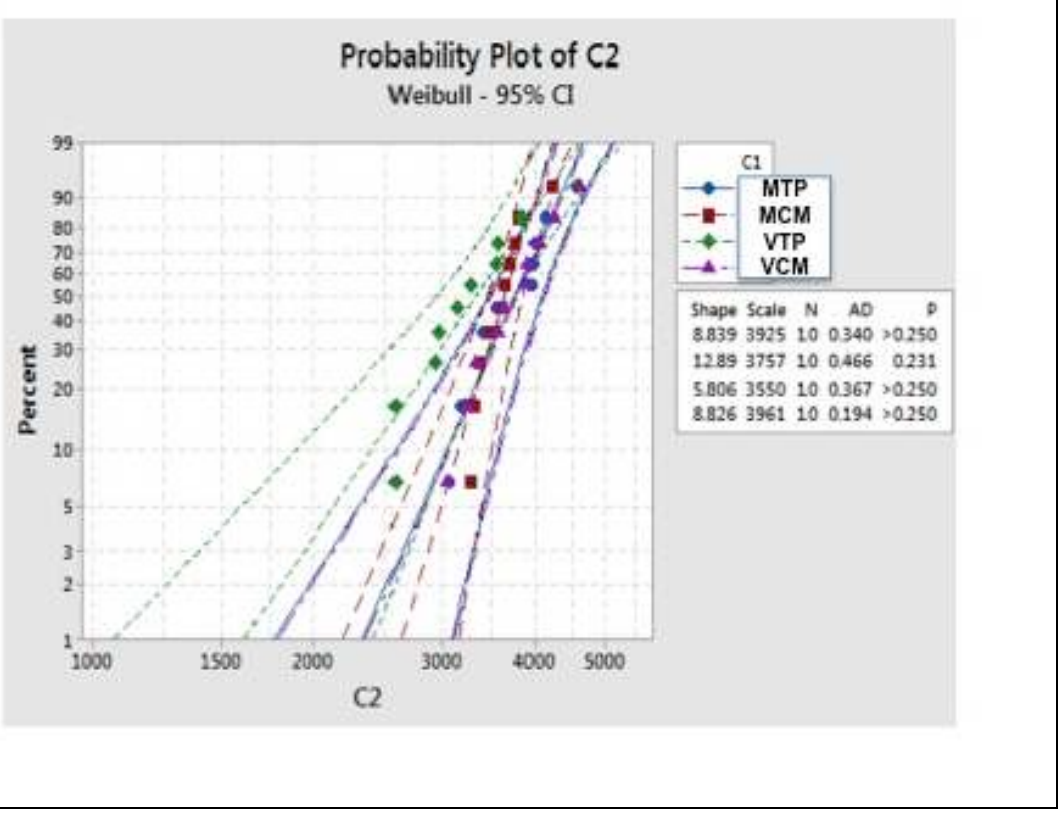

Figure 2 Probability plot with Weibull curves $(95 \% \mathrm{Cl})$ using maximum likelihood estimation, scale and shape values for all groups. See Table 1 for group abbreviations. 

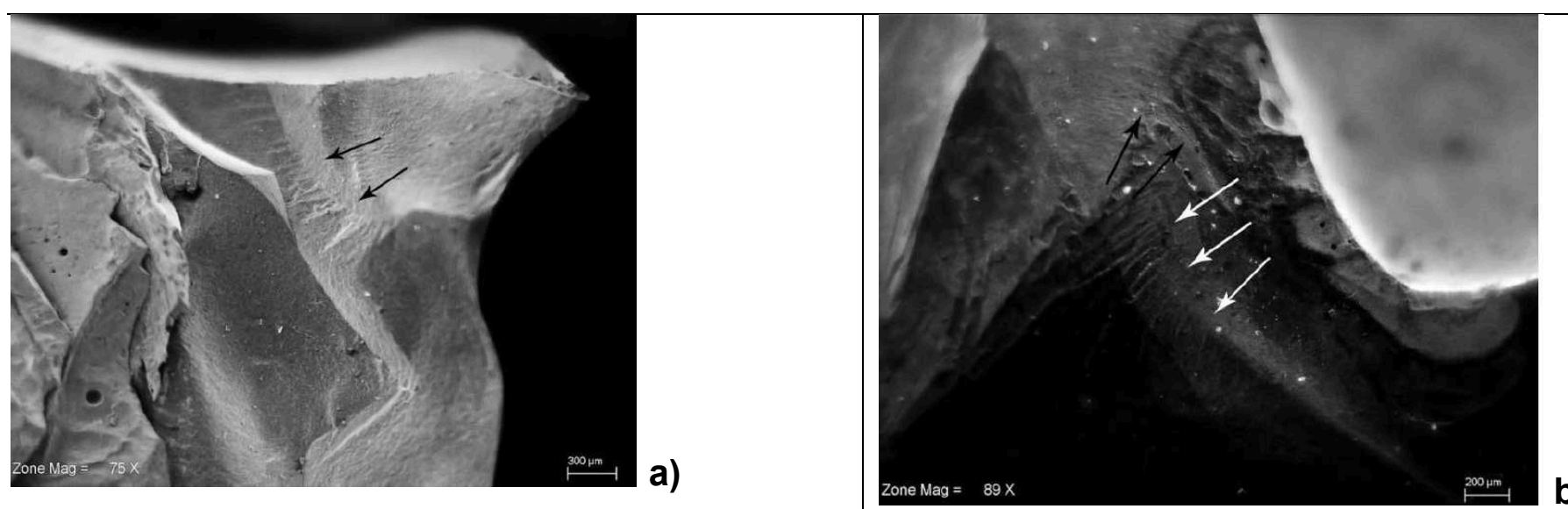

b)

Figures 3a-b SEM images of a) Monolithic lithium disilicate crown after fracture at $x 75$ from the occlusal surface of the veneered crown. The black arrows represent the location and direction of wake hackles and twist hackles. The well-defined concentric lines indicate arrested cracks, b) Veneered lithium disilicate crown after after fracture at x89. The black arrows indicate Wallner lines and white arrows represent the location and direction of wake and twist hackles. 